\title{
Large time behavior of a linear delay differential equation with asymptotically small coefficient
}

Mihály Pituk ${ }^{*}$ and Gergely Röst ${ }^{2}$

Dedicated to Professor Ivan Kiguradze

${ }^{*}$ Correspondence:

pitukm@almos.uni-pannon.hu

${ }^{1}$ Department of Mathematics,

University of Pannonia, P.O. Box 158,

Veszprém, H-8201, Hungary

Full list of author information is

available at the end of the article

\begin{abstract}
The linear delay differential equation $x^{\prime}(t)=p(t) x(t-r), t \geq t_{0}$, is considered, where $r>0$ and the coefficient $p:\left[t_{0}, \infty\right) \rightarrow \mathbb{R}$ is continuous and small in the sense that $\int_{t}^{t+r}|p(s)| d s \rightarrow 0, t \rightarrow \infty$. It is shown that the large time behavior of the solutions can be described in terms of a special solution of the associated formal adjoint equation and the initial data. In the special case of the Dickman-de Bruijn equation, $x^{\prime}(t)=-\frac{x(t-1)}{t}, t \geq 1$, our result yields an explicit asymptotic representation of the solutions as $t \rightarrow \infty$.

MSC: 34K06; 34K25; 11A51
\end{abstract}

Keywords: delay differential equation; formal adjoint equation; Dickman-de Bruijn equation; asymptotic behavior

\section{Introduction}

The linear scalar delay differential equation

$$
x^{\prime}(t)=-\frac{x(t-1)}{t}, \quad t \geq 1
$$

plays a significant role in analytic number theory. Its special solution $x:[0, \infty) \rightarrow \mathbb{R}$ with initial values $x(t)=1$ for $t \in[0,1]$ is called the Dickman-de Bruijn function. The Dickmande Bruijn function was first studied by actuary Dickman [1] and later by de Bruijn [2, 3] to estimate the proportion of smooth numbers up to a given bound. In [4] (see also [5]) van der Lune proposed some problems regarding the solutions of (1.1). The solutions to these problems, given by Bierkens, appeared in [6]. Suppose that $\phi:[0,1] \rightarrow \mathbb{R}$ is a continuous function and let $x=x^{\phi}$ denote the unique solution of (1.1) with initial values

$$
x(t)=\phi(t), \quad 0 \leq t \leq 1 .
$$

Among others, it was shown that if the limit

$$
c(\phi)=\lim _{t \rightarrow \infty}\left[t x^{\phi}(t)\right]
$$

\section{Springer}

(02014 Pituk and Röst; licensee Springer. This is an Open Access article distributed under the terms of the Creative Commons Attribution License (http://creativecommons.org/licenses/by/2.0), which permits unrestricted use, distribution, and reproduction in any medium, provided the original work is properly cited. 
exists and is finite, then its value is given by

$$
c(\phi)=\phi(1)-\int_{0}^{1} \phi(t) d t .
$$

We emphasize that the solution presented in [6] does not imply the existence of the limit (1.3).

Our aim in this paper is twofold. First, we give an alternative proof of the limit relation (1.4) including the existence of the limit in (1.3). Second, we will show that the above result on the Dickman-de Bruijn equation (1.1) can be extended to the general linear equation

$$
x^{\prime}(t)=p(t) x(t-r), \quad t \geq t_{0}
$$

where $r>0, t_{0} \in \mathbb{R}$ and $p:\left[t_{0}, \infty\right) \rightarrow \mathbb{R}$ is a continuous function such that

$$
\int_{t}^{t+r}|p(s)| d s \rightarrow 0 \quad \text { as } t \rightarrow \infty .
$$

Our main result (see Theorem 3.3 below) provides an asymptotic description of the solutions of (1.5). The asymptotic formula is given in terms of the initial data and a special solution of the associated formal adjoint equation

$$
y^{\prime}(t)=-p(t+r) y(t+r), \quad t \geq t_{0}
$$

The special solution of (1.7) is eventually positive, it has bounded growth and it is unique up to a constant multiple.

The large time behavior of the solutions of (1.1) is discussed in Section 2 and our general result on the asymptotic description of the solutions (1.5) is presented in Section 3.

\section{Large time behavior of the Dickman-de Bruijn equation}

In this section, we prove the existence of the limit (1.3) for the solutions of (1.1).

Theorem 2.1 Suppose that $\phi:[0,1] \rightarrow \mathbb{R}$ is a continuous function and let $x^{\phi}$ be the unique solution of the initial value problem (1.1) and (1.2). Then

$$
\lim _{t \rightarrow \infty}\left[t x^{\phi}(t)\right]=\phi(1)-\int_{0}^{1} \phi(t) d t .
$$

The proof of Theorem 2.1 will be based on the identity

$$
t x^{\phi}(t)-\int_{t-1}^{t} x^{\phi}(s) d s=c(\phi), \quad t \geq 1
$$

where $c(\phi)$ is the constant given by (1.4). Using (1.1), it is easily shown that the derivative of the function on the left-hand side of $(2.2)$ is equal to 0 identically on $(1, \infty)$. This, together with (1.4), implies (2.2). Since the proof is straightforward, we omit it.

Now we can give a simple short proof of Theorem 2.1. 
Proof of Theorem 2.1 Write $x^{\phi}=x$ for brevity. First we show that

$$
M=\sup _{t \geq 1}[t|x(t)|]<\infty .
$$

Define

$$
u(t)=t x(t), \quad t \geq 0 .
$$

Then (2.2) can be written in the form

$$
u(t)=c+\int_{t-1}^{t} \frac{u(s)}{s} d s, \quad t \geq 1
$$

where $c=c(\phi)$. From this, we find for $t \geq 2$,

$$
|u(t)| \leq|c|+\int_{t-1}^{t} \frac{|u(s)|}{s} d s \leq|c|+\int_{1}^{t} \frac{|u(s)|}{s} d s=K+\int_{2}^{t} \frac{|u(s)|}{s} d s,
$$

where

$$
K=|c|+\int_{1}^{2} \frac{|u(s)|}{s} d s
$$

From the last inequality, by the application of Gronwall's lemma (see, e.g., [7, Chapter 1, Lemma 3.1]), we conclude that

$$
|u(t)| \leq K \exp \left(\int_{2}^{t} \frac{1}{s} d s\right)=\frac{K t}{2}, \quad t \geq 2
$$

Hence

$$
\sup _{t \geq 2} \frac{|u(t)|}{t} \leq \frac{K}{2}
$$

From this and (2.5), we find for $t \geq 3$,

$$
|u(t)| \leq|c|+\int_{t-1}^{t} \frac{|u(s)|}{s} d s \leq|c|+\frac{K}{2} .
$$

In view of (2.4), this implies (2.3).

From (2.2) and (2.3), we obtain for $t \geq 2$,

$$
|t x(t)-c|=\left|\int_{t-1}^{t} x(s) d s\right| \leq \int_{t-1}^{t}|x(s)| d s \leq \int_{t-1}^{t} \frac{M}{s} d s \leq \frac{M}{t-1}
$$

Letting $t \rightarrow \infty$ in the last inequality, we obtain

$$
\lim _{t \rightarrow \infty}[t x(t)-c]=0
$$

which is equivalent to the limit relation (2.1). 
We remark that the existence of the limit in (1.3) can also be deduced from the results by Győri and the first author (see [8, Theorem 3.3] and its proof) and by Diblík (see [9, Theorem 18 and Example 20]). However, the above results cannot be used to compute the value of the limit explicitly in terms of the initial data.

\section{Main result}

In this section, we present our main result on the large time behavior of the solutions of (1.5). First we show that under the smallness condition (1.6) the formal adjoint equation has an eventually positive solution with bounded growth.

Theorem 3.1 Suppose condition (1.6) holds. Then (1.7) has a solution $y:\left[t_{0}, \infty\right) \rightarrow \mathbb{R}$ which is positive for all large $t$ and such that

$$
\limsup _{t \rightarrow \infty} \frac{y(t+r)}{y(t)}<\infty
$$

We will prove Theorem 3.1 by applying a technique known from the oscillation theory of delay differential equations (see [10, Section 2.3]).

Proof Let $p_{+}$and $p_{-}$denote the positive part and the negative part of $p$, respectively, defined by

$$
p_{ \pm}(t)=\max \{0, \pm p(t)\}, \quad t \geq t_{0}
$$

Since $0 \leq p_{-} \leq|p|$, by virtue of (1.6), there exists $t_{1}>t_{0}$ such that

$$
\int_{t}^{t+r} p_{-}(s) d s \leq \frac{1}{e}, \quad t \geq t_{1}
$$

Let $C\left(\left[t_{1}, \infty\right), \mathbb{R}\right)$ be the space of continuous functions mapping $\left[t_{1}, \infty\right)$ into $\mathbb{R}$ with the topology of uniform convergence on compact subsets of $\left[t_{1}, \infty\right)$. Let $\Omega$ denote the set of functions from $C\left(\left[t_{1}, \infty\right), \mathbb{R}\right)$ which satisfy the system of inequalities

$$
\exp \left(-e \int_{t_{1}}^{t} p_{+}(s+r) d s\right) \leq y(t) \leq \exp \left(e \int_{t_{1}}^{t} p_{-}(s+r) d s\right), \quad t \geq t_{1},
$$

and

$$
\frac{y(t+r)}{y(t)} \leq e, \quad t \geq t_{1}
$$

Clearly, $\Omega$ is a nonempty, closed and convex subset of $C\left(\left[t_{1}, \infty\right), \mathbb{R}\right)$. Define the operator $F: \Omega \rightarrow C\left(\left[t_{1}, \infty\right), \mathbb{R}\right)$ by

$$
F(y)(t)=\exp \left(-\int_{t_{1}}^{t} \frac{p(s+r) y(s+r)}{y(s)} d s\right), \quad t \geq t_{1}, y \in \Omega
$$

It is easily verified that $F$ is continuous and $F(\Omega) \subset \Omega$. Furthermore, the functions from $F(\Omega)$ are uniformly bounded and equicontinuous on each compact subinterval of $\left[t_{1}, \infty\right)$. 
Therefore, by the Arzela-Ascoli theorem, the closure of $F(\Omega)$ is compact in $C\left(\left[t_{1}, \infty\right), \mathbb{R}\right)$. By the application of the Schauder-Tychonoff fixed point theorem, we conclude that there exists $y \in \Omega$ such that $F(y)=y$. It is easily seen that this fixed point $y$ is a solution of (1.7) on $\left[t_{1}, \infty\right)$ with property (3.1). Clearly, the solution $y:\left[t_{1}, \infty\right) \rightarrow \mathbb{R}$ can be extended backward to all $t \in\left[t_{0}, t_{1}\right)$ by the method of steps.

It should be noted that under the smallness condition (1.6), (1.7) may have a positive solution which does not satisfy condition (3.1). Indeed, the equation

$$
y^{\prime}(t)=2 t e^{-2 t-1} y(t+1), \quad t \geq 0,
$$

a special case of (1.7) when $r=1, t_{0}=0$ and $p(t)=-2(t-1) e^{-2 t+1}$, has the positive solution $y(t)=e^{t^{2}}$ for which the ratio $y(t+1) / y(t)=e^{2 t+1}$ is unbounded as $t \rightarrow \infty$.

In the next theorem, we show that up to a constant multiple the special solution of (1.7) described in Theorem 3.1 is unique.

Theorem 3.2 Suppose condition (1.6) holds. Let $y_{1}$ and $y_{2}$ be eventually positive solutions of (1.7) satisfying condition (3.1). Then $y_{2}$ is a constant multiple of $y_{1}$.

Proof We begin with two simple observations. First, if $y$ is a solution of (1.7), then

$$
y(t)=y\left(t_{1}\right)-\int_{t_{1}}^{t} p(s+r) y(s+r) d s \quad \text { whenever } t \geq t_{1} \geq t_{0} .
$$

Second, if $y$ is a solution of (1.7) which is positive on $[T, \infty)$ for some $T>t_{0}$ and satisfies condition (3.1), then

$$
y(t) \leq y\left(t_{1}\right) \exp \left(M \int_{t_{1}}^{t}|p(s+r)| d s\right) \quad \text { whenever } t \geq t_{1} \geq T,
$$

where $M$ is an arbitrary constant such that

$$
M \geq \sup _{t \geq T} \frac{y(t+r)}{y(t)} .
$$

Indeed, from (1.7) we find for $t \geq T$,

$$
y^{\prime}(t)=-p(t+r) \frac{y(t+r)}{y(t)} y(t) .
$$

Hence

$$
y(t)=y\left(t_{1}\right) \exp \left(-\int_{t_{1}}^{t} p(s+r) \frac{y(s+r)}{y(s)} d s\right), \quad t \geq t_{1} \geq T .
$$

This, together with (3.4), implies (3.3).

By assumptions, there exists $T>t_{0}$ such that both solutions $y_{1}$ and $y_{2}$ are positive on $[T, \infty)$ and satisfy condition (3.1). As noted before (see (3.3)), if $M>1$ is sufficiently large, 
then

$$
y_{j}(t) \leq y_{j}\left(t_{1}\right) \exp \left(M \int_{t_{1}}^{t}|p(s+r)| d s\right) \quad \text { whenever } t \geq t_{1} \geq T, j=1,2 .
$$

Since $M>1$, if $q>0$ is sufficiently small, then $e^{q M}<M$. By virtue of (1.6), there exists $t_{1}>T$ such that

$$
\int_{t}^{t+r}|p(s)| d s<q, \quad t \geq t_{1}
$$

We will show that $y_{2}(t)=c y_{1}(t)$ for all $t \geq t_{1}$, where $c=y_{2}\left(t_{1}\right) / y_{1}\left(t_{1}\right)$. In view of the linearity of (1.7), the function $y_{3}=c y_{1}$ is a solution of (1.7) and, by virtue of (3.5), the quantity

$$
S=\sup _{t \geq t_{1}}\left[\left|y_{2}(t)-y_{3}(t)\right| \exp \left(-M \int_{t_{1}}^{t}|p(s+r)| d s\right)\right]
$$

is finite. Applying (3.2) to both solutions $y_{2}$ and $y_{3}$ of (1.7) and taking into account that $y_{3}\left(t_{1}\right)=y_{2}\left(t_{1}\right)$, we obtain, for $t \geq t_{1}$,

$$
\begin{aligned}
\left|y_{2}(t)-y_{3}(t)\right| & =\left|\int_{t_{1}}^{t} p(s+r)\left(y_{2}(s+r)-y_{3}(s+r)\right) d s\right| \\
& \leq \int_{t_{1}}^{t}|p(s+r)|\left|y_{2}(s+r)-y_{3}(s+r)\right| d s \\
& \leq S \int_{t_{1}}^{t}|p(s+r)| \exp \left(M \int_{t_{1}}^{s+r}|p(u+r)| d u\right) d s \\
& =S \int_{t_{1}}^{t}|p(s+r)| \exp \left(M \int_{t_{1}}^{s}|p(u+r)| d u\right) \exp \left(M \int_{s}^{s+r}|p(u+r)| d u\right) d s \\
& \leq S e^{q M} \int_{t_{1}}^{t}|p(s+r)| \exp \left(M \int_{t_{1}}^{s}|p(u+r)| d u\right) d s \\
& =S e^{q M}\left[\frac{1}{M} \exp \left(M \int_{t_{1}}^{s}|p(u+r)| d u\right)\right]_{t_{1}}^{t} \\
& \leq S \frac{e^{q M}}{M} \exp \left(M \int_{t_{1}}^{t}|p(u+r)| d u\right),
\end{aligned}
$$

where the last but one inequality is a consequence of (3.6). From the last inequality, we obtain

$$
\left|y_{2}(t)-y_{3}(t)\right| \exp \left(-M \int_{t_{1}}^{t}|p(u+r)| d u\right) \leq S \frac{e^{q M}}{M}, \quad t \geq t_{1} .
$$

Hence

$$
S \leq \frac{e^{q M}}{M} S
$$

Since $e^{q M}<M$, this implies that $S=0$ and therefore $y_{2}(t)=y_{3}(t)=c y_{1}(t)$ for all $t \in\left[t_{1}, \infty\right)$. Finally, by the uniqueness of the backward continuation of the solutions of (1.7), we conclude that $y_{2}(t)=c y_{1}(t)$ for all $t \in\left[t_{0}, \infty\right)$. 
Now we can formulate our main result about the large time behavior of the solutions of (1.5).

Theorem 3.3 Suppose condition (1.6) holds. Let $x^{\phi}$ denote the solution of (1.5) with initial data

$$
x(t)=\phi(t), \quad t_{0}-r \leq t \leq t_{0},
$$

where $\phi:\left[t_{0}-r, t_{0}\right] \rightarrow \mathbb{R}$ is a continuous function. Then

$$
x^{\phi}(t)=\frac{1}{y(t)}(c(\phi)+o(1)), \quad t \rightarrow \infty
$$

where $y$ is any eventually positive solution of (1.7) satisfying (3.1) and $c(\phi)$ is a constant given by

$$
c(\phi)=\phi\left(t_{0}\right) y\left(t_{0}\right)+\int_{t_{0}-r}^{t_{0}} p(s+r) \phi(s) y(s+r) d s .
$$

As shown in Theorem 3.2, the special solution $y$ of (1.7) in the asymptotic relation (3.8) is unique up to a constant multiple. Thus, (3.8) gives the same asymptotic representation independently of the choice of $y$.

Theorem 3.3 is a generalization of Theorem 2.1 to (1.5). Indeed, in the special case $r=1$, $t_{0}=1$ and $p(t)=-t^{-1} ;(1.5)$ reduces to the Dickman-de Bruijn equation (1.1). Its formal adjoint equation

$$
y^{\prime}(t)=\frac{y(t+1)}{t+1}, \quad t \geq 1
$$

has the positive solution $y(t)=t$ satisfying condition (3.1). Therefore, Theorem 3.3 applies and its conclusion reduces to the limit relation (1.3).

For qualitative results similar to Theorem 3.3, see $[8,9,11,12]$ and the references therein. The proof of Theorem 3.3 will be based on the well-known duality between the solutions of a linear delay differential equation and its formal adjoint equation (see [7, Section 6.3]). Namely,

$$
x(t) y(t)+\int_{t}^{t+r} p(s) x(s-r) y(s) d s=\mathrm{constant}
$$

for $t \geq t_{0}$ whenever $x$ and $y$ are solutions of (1.5) and (1.7), respectively. We will also need the following simple lemma.

Lemma 3.4 Let $c \in \mathbb{R}$ and suppose that $a:\left[t_{0}, \infty\right) \rightarrow \mathbb{R}$ is a continuous function such that

$$
\int_{t}^{t+r}|a(s)| d s \rightarrow 0, \quad t \rightarrow \infty
$$

Then every continuous solution of the integral equation

$$
z(t)=c+\int_{t}^{t+r} a(s) z(s-r) d s, \quad t \geq t_{0},
$$

converges to $c$ as $t \rightarrow \infty$. 
Proof Let $q \in(0,1)$. By virtue of (3.11), there exists $T>t_{0}$ such that

$$
\int_{t}^{t+r}|a(s)| d s<q, \quad t \geq T
$$

Define

$$
M=\max _{T-r \leq t \leq T}|z(t)|
$$

Choose a constant $K$ such that

$$
K>\max \left\{M,|c|(1-q)^{-1}\right\} .
$$

Clearly, $|z(t)| \leq M<K$ for $t \in[T-r, T]$ and we claim that

$$
|z(t)|<K \quad \text { for all } t \geq T-r .
$$

Otherwise, there exists $t_{1}>T$ such that

$$
|z(t)|<K \quad \text { for } t \in\left[T-r, t_{1}\right) \text { and }\left|z\left(t_{1}\right)\right|=K \text {. }
$$

From this and (3.12), we find that

$$
\begin{aligned}
K & =\left|z\left(t_{1}\right)\right| \leq|c|+\int_{t_{1}}^{t_{1}+r}|a(s)||z(s-r)| d s \\
& \leq|c|+K \int_{t_{1}}^{t_{1}+r}|a(s)| d s \leq|c|+K q,
\end{aligned}
$$

the last inequality being a consequence of (3.13). Hence $K \leq|c|(1-q)^{-1}$, contradicting (3.14). Thus, (3.15) holds.

From (3.12) and (3.15), we find for $t \geq T$,

$$
|z(t)-c| \leq \int_{t}^{t+r}|a(s)||z(s-r)| d s \leq K \int_{t}^{t+r}|a(s)| d s .
$$

Letting $t \rightarrow \infty$ in the last inequality and using (3.11), we conclude that $z(t) \rightarrow c$ as $t \rightarrow \infty$.

Now we are in a position to give a proof of Theorem 3.3.

Proof of Theorem 3.3 Write $x^{\phi}=x$ for brevity and let $y$ be a solution of (1.7) which is positive on $\left[t_{1}, \infty\right)$ for some $t_{1}>t_{0}$ and satisfies condition (3.1). By virtue of (3.7) and (3.10), we have

$$
x(t) y(t)+\int_{t}^{t+r} p(s) x(s-r) y(s) d s=c(\phi)
$$

for $t \geq t_{0}$ with $c(\phi)$ as in (3.9). If we let

$$
z(t)=x(t) y(t), \quad t \geq t_{0},
$$


then (3.16) can be written in the form (3.12) with

$$
c=c(\phi), \quad a(t)=-p(t) \frac{y(t)}{y(t-r)}, \quad t \geq t_{1}+r
$$

and $t_{0}$ replaced with $t_{1}+r$. Clearly, conditions (1.6) and (3.1) imply that assumption (3.11) of Lemma 3.4 is satisfied. By the application of Lemma 3.4, we conclude that

$$
\lim _{t \rightarrow \infty} z(t)=\lim _{t \rightarrow \infty}[x(t) y(t)]=c
$$

which is only a reformulation of the limit relation (3.8).

Finally, we remark that applying a transformation technique described in [13] and [14], Theorem 3.3 can possibly be extended to a class of equations with time-varying delays.

Competing interests

The authors declare that they have no competing interests.

\section{Authors' contributions}

All authors contributed equally to the writing of this paper. All authors read and approved the final manuscript.

\section{Author details}

${ }^{1}$ Department of Mathematics, University of Pannonia, P.O. Box 158, Veszprém, H-8201, Hungary. ${ }^{2}$ Bolyai Institute, University of Szeged, Aradi vértanúk tere 1, Szeged, H-6720, Hungary.

\section{Acknowledgements}

M Pituk was supported in part by the Hungarian National Foundation for Scientific Research (OTKA) Grant No. K101217. G Röst was supported in part by European Union and co-funded by the European Social Fund under the project 'Telemedicine focused research activities on the field of Mathematics, Informatics and Medical sciences' of project number TÁMOP-4.2.2.A-11/1/KONV-2012-0073, ERC Starting Grant No. 259559 and OTKA K109782.

Received: 4 February 2014 Accepted: 15 April 2014 Published: 14 May 2014

\section{References}

1. Dickman, K: On the frequency of numbers containing prime factors of a certain relative magnitude. Ark. Mat. Astron. Fys. 22A(10), 1-14 (1930)

2. de Bruijn, NG: On the number of positive integers $\leq x$ and free of prime factors $>y$. I. Indag. Math. 13, 50-60 (1951)

3. de Bruijn, NG: On the number of positive integers $\leq x$ and free of prime factors $>y$. II. Indag. Math. 2, 239-247 (1966)

4. Nieuw Archief voor Wiskunde, Problem Section, 4/14 no. 3 Nov. 1996, p.429

5. Nieuw Archief voor Wiskunde, Problem Section, 5/9 no. 2 June 2008, p.232

6. Nieuw Archief voor Wiskunde, Problem Section, 5/11 no. 1 March 2010, p.76

7. Hale, JK: Theory of Functional Differential Equations. Springer, New York (1977)

8. Győri, I, Pituk, M: Stability criteria for linear delay differential equations. Differ. Integral Equ. 10, $841-852$ (1997)

9. Diblík, J: Behaviour of solutions of linear differential equations with delay. Arch. Math. 34, 31-47 (1998)

10. Győri, I, Ladas, G: Oscillation Theory of Delay Differential Equations with Applications. Oxford University Press, New York (1991)

11. Arino, O, Pituk, M: More on linear differential systems with small delays. J. Differ. Equ. 170, 381-407 (2001)

12. Diblík, J, Rưžičková, M: Asymptotic behavior of solutions and positive solutions of differential delayed equations. Funct. Differ. Equ. 14, 83-105 (2007)

13. Čermák, J: Note on canonical forms for functional differential equations. Math. Pannon. 11, 29-39 (2000)

14. Neuman, F: On transformations of differential equations and systems with deviating argument. Czechoslov. Math. J. 31, 87-90 (1981)

10.1186/1687-2770-2014-114

Cite this article as: Pituk and Röst: Large time behavior of a linear delay differential equation with asymptotically small coefficient. Boundary Value Problems 2014, 2014:114 